\title{
Introduction to the Issue on Interdiffused Quantum-Well Materials and Devices
}

T HIS ISSUE of IEEE JOURNAL OF SELECTED TOPICS IN QUANTUM ELECTRONICS underscores the latest trends of developments in the fabrication of photonic integrated circuits (PIC's), which can be an attractive means to fully utilize the enormous bandwidth potential of optical communications. Beyond all doubt, a practical technique for integrating both active and passive photonic devices, such as tunable lasers, modulators, and photodetectors, will produce revolutionary breakthroughs in areas of information processing, distribution, and manipulation. However, for some time now, the realization of such a Utopian vision has been hampered by the magnitude of the technological challenges involved. Substantial efforts on the part of a considerable number of research groups worldwide, in response to skyrocketing demand for multiplewavelength optical networks driven by the exponential growth of the Internet, and, in particular, the World Wide Web, have not produced expected results.

Practical PIC's mandate low manufacturing cost and high reliability, that is, low operating cost, even at the expense of a somewhat reduced performance. The realization of such a fabrication scheme must establish the most fundamental condition for integrating optoelectronic devices of differing functionalities monolithically, which is material bandgap/wavelength compatibility among various optoelectronic devices. The mere existence of a number of techniques which meets this requirement by no means implies that a complete solution has been found, as these techniques all have their own strengths and limitations.

The simple and practical technique highlighted in this volume not only provides an elegant solution for monolithically integrating optoelectronic devices, but can also serve to enhance some devices' performance. To be more specific, the technique makes use of spatially selective modification of quantum-well (QW) shapes after standard epitaxial growth, which in turn modifies the QW bandgap energy. Also referred to as quantum-well mixing or intermixing (QWI), an interdiffused or diffused quantum well (DFQW) is a nonsquare QW produced by the interdiffusion of constituent atoms across the well-barrier heterointerface. Ion-implantation-induced QW composition disordering (IID) and impurity-free vacancy disordering (IFVD) are but a few examples of the selective techniques available. In fact, as demonstrated by the pioneering work of Thornton et al., who integrated a gain region, an interconnecting waveguide and an electroabsorption modulator, there is no longer any dispute that QWI can be used to fabricate moderately complex PIC's.

Having no deleterious effects on the optical, the electrical, or the lifetime properties of optoelectronic devices, bandgap modification is a simple yet powerful technique which

Publisher Item Identifier S 1077-260X(98)07745-4. possesses certain surprising advantages, particularly in the reduction of polarization sensitivity of some devices and in the enhancement of reflectance changes. Its straightforward implementation makes it possible to simplify the fabrication of many complex PIC's, thereby rendering them practical enough for commercial utilization. Using this technology, the bandgap and optical properties of QW materials [(Al)GaInP-GaAs, AlGaAs-GaAs, InGaAs-GaAs, InGa(Al)As-InP, and InGaAs(P)-InP] can be modified through interdiffusion (intermixing), and the tuning ability of these properties can be exploited in such integrated device structures as waveguide detectors and laser-modulators. It is then possible to fabricate a multisection integrated structure for wide bandwidth and multiwavelength applications, of which the use of wavelength-division multiplexing (WDM) in high bit-rate optical communication systems is a case in point.

Indeed, recent months have witnessed much progress in QWI technology. A number of new techniques have been developed to selectively enhance QW intermixing, namely anodic-oxide-induced intermixing, laser-assisted disordering and proton irradiation. As for elucidating diffusion mechanisms, computer simulations of double crystal X-ray diffraction (DCXRD) curves for annealed multiple QW structures have proven useful. In addition, much effort has been put into expanding device applications. For instance, the incorporation of waveguides into vertical cavity devices is a relatively novel concept.

The state of the art of this highly interesting and often compelling technology has been outlined in the topics covered by the invited papers in this issue. To begin, Holonyak, Jr. traces the entire course of development of impurity-induced layer disordering of $\mathrm{QW}$ heterostructures. The dependence of atomic diffusion in GaAs upon experimental design is then explored by Cohen, followed by the work of Jagadish's group on anodic-oxide induced intemixing in GaAs-AlGaAs QW's and quantum-wire structures. Apart from this, the work of Coldren's group on interdiffused QW's for lateral carrier confinement in VCSEL's provides some very good insights concerning the use of QWI. Issues related to the production of semiconductor lasers using diffused QW structures are dealt with by $\mathrm{Yu}$ and Li. More specifically, the topic of monolithic optoelectronic device integration has been touched upon by three different groups of researchers. Forchel and Reithmaier advocate the use of focused ion beam implantation induced thermal QWI; Charbonneau et al. discuss the viability of using ion implantation; and Marsh's group examines the pros and cons of the universal damage enhanced QWI technique.

All things considered, QWI has provided a very enticing vision for the future of optoelectronics. Not only have advances in its development improved the performance, the reliability and the integration of existing optical components, 
but they have also led to the development of novel optical functions. Undoubtedly, this has strong implications for the realization of a tunable and high-performance device, such as laser, electrooptic modulator, vertical-cavity waveguide and their monolithic integration in building PIC's.

We would like to take this opportunity to express our genuine appreciation for all the reviewers involved, without whose wholehearted efforts this issue could not have been brought to fruition. As a general rule, the IEEE JSTQE has a single deadline for all papers, which means that papers not reviewed in a timely manner will not be published. In addition, apologies must also be given to authors whose papers were not able to meet the deadline. We would also like to thank Ms. F. Jetko, the Publications Administrator of the LEOS Editorial Office, for her enthusiastic help in coordinating overall publication issues.

\author{
E. HERBERT LI, Guest Editor \\ Department of Electrical and Electronic Engineering \\ University of Hong Kong \\ Hong Kong
}

\author{
EMIL S. KoTELES, Guest Editor \\ Institute for Microstructural Sciences \\ National Research Council \\ Ottawa, ON, K1A 0R6, Canada
}

\author{
JOHN H. MARSH, Guest Editor \\ Department of Electronics and Electrical Engineering \\ University of Glasgow \\ Glasgow G12 8QQ, Scotland, U.K.
}

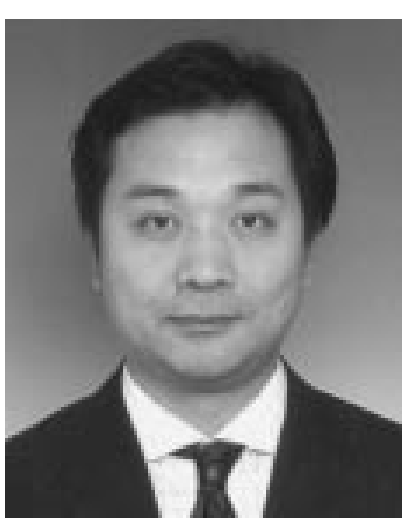

E. Herbert Li (SM'95) received the M.Phil. degree in applied mathematics and electronic engineering from the University of Hong Kong, and the Ph.D. degree in electronic engineering from the University of Surrey, Surrey, U.K. His M.Phil thesis was concerned with the modeling of Si-based MOS devices while the doctoral dissertation involved the development of III-V semiconductor based optoelectronic and photonic devices utilizing quantum wells.

He worked for the Kirsten Aeronautical Laboratory, Seattle, WA (1979-1980). He joined K K Engineering Company, Hong Kong, as an Engineer (1981-1986), and Micro Systems, Hong Kong, as a Manager (1986-1988). He was a Member of Faculty at the City University of Hong Kong (1988-1990). He joined the National Ion Implantation Facility at the Department of Electrical and Electronic Engineering, University of Surrey, U.K., as a Research Project Leader (1990-1993) where he is now a Senior Visiting Fellow. He is a Member of Faculty and Leader of the Optoelectronics Group, which consists of a group of ten researchers, at the Department of Electronics and Electrical Engineering, University of Hong Kong (1994-present). He is a Visiting Scholar at Harvard University, Cambridge, MA (1997-1998), a Guest Professor of the Institute of Semiconductors, Shandong University, China (1997-present), and also holds Visiting Faculty appointments at the University of Waterloo, Waterloo, Canada, and Tsinghua University, China. His earlier research activities are involved with the modeling and simulation of miniaturized size effect in Si-based MOSFET's, and the development of computer aided devices and systems in rehabilitation engineering. Recently, he became interested in the area of III-V compound semiconductor devices, which research has been supported by the U.S. Army, Defense Advanced Research Project Agency (U.S.), SERC (U.K.), and RGC (Hong Kong). His current research interests are concerned with optoelectronic device fabrication, modeling and characterization, interdiffusion modified quantum-well structures for the integration of optoelectronic devices, acousticoptic devices for slow-rate switching applications, photovoltaic devices, nitride-materials-based full-color display technology, and the development of blue bandgap as well as far-IR materials. Since 1990, he has published over 140 international technical papers and five monographs including the selected papers on Quantum Well Mixing and Optoelectronic Device Applications in the SPIE Milestone Series (Bellingham, 1998), Semiconductor Quantum Well Intermixing-Material Properties and Optoelectronics Applications (Gordon \& Breach, 1998), and Intermixed Quantum Wells for Photonics (New York: Wiley).

Prof. Li is a "Distinguished Lecturer" of the IEEE Electron Devices Society (ED-S) since 1997. He is a ED-S representative for the IEEE Technology Policy Council-Research \& Development Committee, a member of the EDS Administrative Committee (AdCom), EDS Meeting Committee, EDS Optoelectronic Devices Technical Committee, and EDS Compound Semiconductor IC Technical Committee. He co-organized the First Chinese Optoelectrionics Workshop in 1994 and served on the technical program committee of the IEEE TENCON'95 on Microelectronics and VLSI in 1995, and on both of the Technical Program and International Advisory Committees of OECC '96, '98 Japan, and '97 Korea. He is an Editor of the International Journal of Optoelectronics (Taylor \& Francis, U.K.). He is also an Honorary Life Advisor of Pi Mu Epsilon (U.S.). He is a Fellow of the Institute of Physics and the Institute of Mathematics and Its Applications (U.K.), as well as a Chartered Engineer, a Chartered Physicist, and a Chartered Mathematician in the U.K. He received the J. Langham Thompson Premium Prize in 1992 from the Institution of Electrical Engineers (U.K.) and the Distinguished Pioneering Projects Award in 1989 from HKCSS (Hong Kong). His biography is published in Who's Who in the World (Marquis, U.S.), Who's Who in Australiasia and the Far East (IBC, U.K.), and Who's Who in Science and Engineering (Marquis, U.S.). 


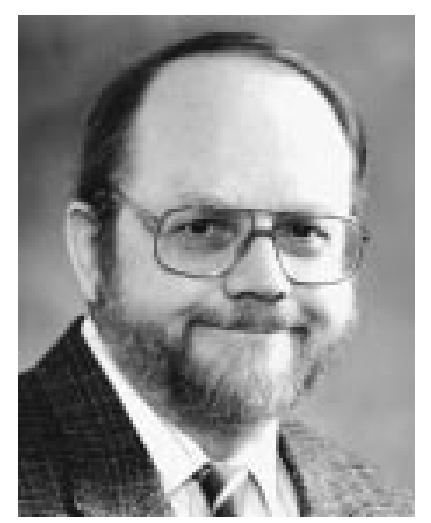

Emil S. Koteles (M'81) received the B.Sc. degree (honors) in physics from Assumption University of Windsor, Windsor, ON, Canada, the M.Sc. degree in physics from the University of Windsor, Windsor, ON, Canada, and the Ph.D. degree in physics from McMaster University, Hamilton, ON, Canada, in 1973. His Ph.D. dissertation dealt with far-infrared absorption processes in InSb.

After post-doctoral work at McMaster expanding his Ph.D. work to other III-V and II-VI compound semiconductors, he was a Guest Scientist at the Max Planck Institute for Solid State Sciences, Stuttgart, Germany, for two and a half years. During his stay, he helped develop a novel technique for measuring exciton-polariton dispersion in bulk semiconductors using resonant Brillouin spectroscopy. After a year at City College, New York, NY, he became a Member of the Technical Staff at GTE Laboratories, Waltham, MA, in 1979. His interests there included far-infrared surface plasmons and optical studies of compound semiconductor bulk and heterostructure layers. In particular, he employed low-temperature photoluminescence (PL) and PL excitation spectroscopies to investigate single and coupled quantum wells and the effects of internal and external perturbations, such as electric fields, temperature, and strain, on them. He also initiated studies on vacancy enhanced quantumwell shape modification as a technique for monolithic integration of optoelectronic components of varying functionalities. He helped popularize the use of ion implantation as a simple technique for quantum-well intermixing. In 1993, he joined the Institute for Microstructural Sciences (IMS) of the National Research Council of Canada in Ottawa, ON, Canada, as a Senior Research Officer. He was made a Principal Research Officer in 1997 and is the Group Leader for Optoelectronics at IMS. His interests now center on optoelectronic devices such as waveguide grating DEMUXes, semiconductor optical amplifiers, modulators, etc., and techniques for monolithically integrating them. He has published over 180 articles in scientific journals and conference proceedings. He has recently written a review chapter on ion implantation enhanced quantum-well intermixing which will be published in Semiconductor Quantum Well Intermixing in 1998. He has given many invited presentations at international conferences and is on the program committee of the 1998 International Conference on Applications of Photonic Technology. He is on the Editorial Board of the International Journal of Optoelectronics.

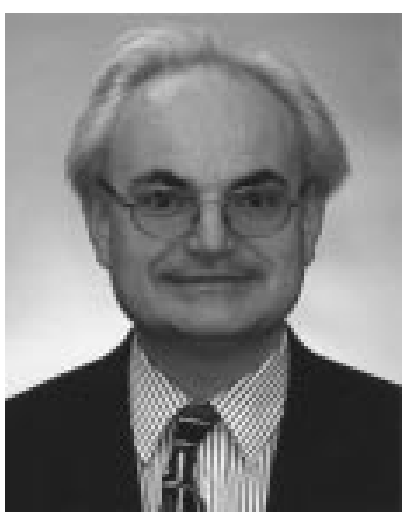

John H. Marsh (M'91-SM'91) was born in Edinburgh, Scotland, U.K., in 1956. He received the B.A. degree in engineering and electrical sciences from the University of Cambridge, Cambridge, U.K., in 1977, the M.Eng. degree in solid-state electronics from the University of Liverpool, Liverpool, U.K., in 1978, and the Ph.D. degree in the LPE growth and electrical transport properties of InGaAsP alloys from Sheffield University, Sheffield, U.K., in 1982.

He is currently a Professor of Optoelectronic Systems in the Department of Electronics and Electrical Engineering at Glasgow University having previously been a Lecturer (1986), Senior Lecturer (1990) and Reader (1994). In 1988-1994, he was a member of the IEE Professional Group Committee concerned with Optical Devices and Systems, and was a corresponding editor of the IEE Electronics and Communication Journal. He was Director of the NATO Advanced Study Institute on Waveguide Optoelectronics, Glasgow, Scotland, U.K., in 1990 and is coeditor of the book of the same name. He is Technical Director of the Department's industrial and commercial interface, Kelvin Nanotechnology (KNT). His research interests are particularly concerned with linear and nonlinear integrated optoelectronic systems. He has developed new integration technologies for photonic integrated circuits based on quantum-well devices and quantum-well intermixing and leads the programme of work at Glasgow on III-V based photonic integrated circuits for high-speed digital optical communications. He is author or coauthor of more than 260 journal and conference papers. He is a member of the Engineering and Physical Sciences Research Council's Electronic and Photonic Technologies College and the Management Panel of Next Generation Laser Diodes. He is a member of the Editorial Board of the International Journal of Optoelectronics.

Dr. Marsh is currently Chair of the Scottish Chapter of the IEEE Lasers and Electro-Optics Society and the Student Counsellor for the IEEE U.K. and Republic of Ireland Student Branch. He is a Fellow of the Institution of Electrical Engineers (FIEE), Fellow of the Royal Society of Arts (FRSA), Senior Member of the IEEE Lasers and Electro-Optics Society, and a member of the British Association for Crystal Growth. 